\title{
Synthesis and Characterization of Genistein Magnetic Molecularly Imprinted Polymers and Its Application in Soy Sauce Products
}

\section{Ziqi Xie}

Beijing University of Technology

Yunjing Luo ( $\square$ luoyj@bjut.edu.cn )

Beijing University of Technology

\section{Zhen Na}

Beijing University of Technology

Wei Zhang

Beijing University of Technology

Yunfei Zong

Beijing University of Technology

\section{Research Article}

Keywords: Genistein, Magnetic nanoparticles, Molecular imprinted polymers, High performance liquid chromatography

Posted Date: August 10th, 2021

DOl: https://doi.org/10.21203/rs.3.rs-774651/v1

License: (c) (i) This work is licensed under a Creative Commons Attribution 4.0 International License. Read Full License 


\section{Synthesis and characterization of genistein magnetic molecularly imprinted}

\section{polymers and its application in soy sauce products}

Ziqi Xie, Yunjing Luo*, Zhen Na, Wei Zhang, and Yunfei Zong

Faculty of Environment and Life, Beijing University of Technology, No.100, Pingleyuan, Chaoyang District, Beijing, 100124, China

Corresponding author: Dr. Yunjing Luo; No.100, Pingleyuan, Chaoyang District, Beijing, 100124, China; E-mail: luoyj@bjut.edu.cn; ORCID: 0000-0002-8915-369X

\section{Abstract}

In this study, a novel method based on genistein magnetic molecularly imprinted polymers (Gen-MMIPs) was developed by surface molecular imprinting technique, in which genistein was used as the template molecule and $\mathrm{Fe}_{3} \mathrm{O}_{4}$ was used as the carrier. The synthesis of Gen-MMIPs were characterized by using of scanning electron microscopy (SEM) and transmission electron microscopy (TEM), which indicated the diameters of Gen-MMIPs were about $500 \mathrm{~nm}$. Through the technique of vibrating sample magnetometer (VSM), the saturation magnetization of Gen-MMIPs were detected as $24.79 \mathrm{emu} / \mathrm{g}$. Fourier transform infrared (FR-IR) spectroscopy showed that polymer groups were on the surface of the magnetic carrier. Adsorption experiment suggested the adsorption capability of Gen-MMIPs to genistein were $1.55 \mathrm{mg} / \mathrm{g}$, and 
the adsorption equilibrium was achieved within $20 \mathrm{~min}$. Gen-MMIPs as dispersive solid-phase extraction adsorbent combined with HPLC was used to selectively separate genistein in soy sauce samples, the recoveries were ranged from $85.7 \%$ to $88.5 \%$ with the relative standard deviations (RSD) less than 5\%, which proved this method can be used for the detection of genistein residues in real samples.

Key words: Genistein; Magnetic nanoparticles; Molecular imprinted polymers; High performance liquid chromatography

Declarations: Not applicable

\section{INTRODUCTION}

Genistein is an important bioactive component, which exist in many soybean plants. Previous reports showed that genistein had a series of pharmacological properties, such as anti-cancerous[1], preventing osteoporosis[2] and reducing postmenopausal problems[3]. However, the content of genistein in real products is extremely low. It is difficult to detect genistein in complex practical samples, which limits its application.

Several methods have been reported to determine genistein, including mass spectrometry[4], liquid chromatography[5, 6], gas chromatography[7], and enzyme- 
linked immunosorbent assay[8]. Although these methods can effectively identify genistein, real samples contain a large number of complex matrices, so that the analysis of genistein requires sample pre-treatment methods. Sample pre-treatment methods currently include solid-phase extraction(SPE)[9], solid-phase microextraction (SPME)[10] liquid-liquid extraction(LLE)[11], and Supercritical fluid extraction (SFE)[12, 13], but these methods led to the low selectivity and sensitivity. Therefore, it is necessary to develop a method with good stability, high selectivity, and high sensitivity to detect genistein in real sample.

Molecularly imprinted polymers (MIPs) have been synthesized as new innovative sorbents[14-16], which possess excellent characteristics for their inexpensive, easy to prepare, excellent reusability and high selectivity[17]. Due to excellent adsorption performance, MIPs have attracted more and more attention[18]. Compared to ordinary MIPs, magnetic molecularly imprinted polymer (MMIPs) has advantages such as larger specific surface area, unique paramagnetism and better specificity and adsorption performance than ordinary polymers[19, 20], synthesis principle is shown in Figure 1(A). MMIPs can be used as an adsorbent for dispersive solid phase extraction, which can be directly dispersed in the sample, the target is extracted and enriched in the solution. After adsorption time reached balance, MMIPs can be quickly separated from 
the solution by applying an external magnetic field[21], which simplifies the experimental operation and reduces the use of organic reagents, thereby achieving the purpose of rapid separation and enrichment of the target[22,23]. Therefore, this study tried to design and synthesize a magnetic molecularly imprinted column that can specifically recognize genistein and establish a detection method for genistein in a complex matrix.

\section{MATERIALS AND METHODS}

\subsection{Reagents and chemicals}

Genistein, daidzein, 4-vinylpyridine (4-VP), azobisisobutyronitrile (AIBN), 3aminopropyltriethoxysilane (APTES), ethylene glycol dimethacrylate (EGDMA) were purchased from Sigma-Aldrich (Steinheim, Germany). 3-trimethoxysilyl-propylmethacrylate (MPS), tetraethoxysilane (TEOS), Iron (III) chloride hexahydrate $\left(\mathrm{FeCl}_{3} \cdot 6 \mathrm{H}_{2} \mathrm{O}\right)$, iron (II) sulfate heptahydrate $\left(\mathrm{FeSO}_{4} \cdot 7 \mathrm{H}_{2} \mathrm{O}\right)$, were purchased from Aladdin (Shanghai, China). Acetonitrile (HPLC grade) was purchased from Merck (Darmstadt, Germany). All other reagents and chemicals were of analytical grade or higher.

\subsection{Apparatus}

The morphologies of samples were measured by scanning electron microscopy (S- 
4800, Hitachi, Japan) and transmission electron microscope (Tecnai G2 F30, FEI, USA).

The groups on the surface of the polymers were detected using a Fourier transform infrared spectrometer (FT-IR, Nicolet 6700, ThermoFisher, USA). The magnetic properties were measured using a vibrating sample magnetometer (VSM, MPMS-3, Quantum Design, USA) at room temperature.

\subsection{HPLC conditions}

Chromatographic analysis was performed on HPLC-DAD system (Waters 2695, Waters Technologies, USA) with a C18 column (SunFire RM C18, 4.6×150 mm, $5 \mu \mathrm{m}$ ). The mobile phase was acetonitrile/ $0.1 \%$ phosphoric acid aqueous solution $(5: 5, \mathrm{~V} / \mathrm{V})$ at a flowrate of $1.0 \mathrm{~mL} / \mathrm{min}$ at $25^{\circ} \mathrm{C}$. Spectra were monitored at $260 \mathrm{~nm}$.

\subsection{Preparation process}

\subsubsection{Synthesis of $\mathrm{Fe}_{3} \mathrm{O}_{4}$ nanoparticles}

$\mathrm{Fe}_{3} \mathrm{O}_{4}$ nanoparticles were synthesized via co-precipitation[24, 25]. $\mathrm{FeCl}_{2} \cdot 4 \mathrm{H}_{2} \mathrm{O}$ $(0.88 \mathrm{~g})$ and $\mathrm{FeCl}_{3} \cdot 6 \mathrm{H}_{2} \mathrm{O}(2.35 \mathrm{~g})$ were dissolved in $40 \mathrm{~mL}$ of ultrapure water. The mixture was heated to $70^{\circ} \mathrm{C}$, and $20 \mathrm{~mL}$ of $25 \%$ ammonia was added into it. Then, the reaction maintained under constant stirring at $70^{\circ} \mathrm{C}$ for $30 \mathrm{~min}$. The products were collected by an external magnetic field and washed five times with ethanol. Finally, the products dried in a vacuum at $60{ }^{\circ} \mathrm{C}$ for $12 \mathrm{~h}$. 


\subsubsection{Synthesis of $\mathrm{Fe}_{3} \mathrm{O}_{4} @ \mathrm{SiO}_{2}$}

$\mathrm{Fe}_{3} \mathrm{O}_{4}$ nanoparticles $(500 \mathrm{mg})$ were dispersed into $250 \mathrm{~mL}$ ethanol/water $(8: 2$, $\mathrm{V} / \mathrm{V}$ ) under ultrasonication for $30 \mathrm{~min}$. Thereafter, $10 \mathrm{~mL}$ of $25 \%$ ammonia and $10 \mathrm{~mL}$ of TEOS were added sequentially under nitrogen protection. Then, the solution was stirred at $250 \mathrm{rpm}$ for $12 \mathrm{~h}$ at room temperature. The resultant nanoparticles were washed three times with ethyl alcohol and water after isolated by an external magnetic. Finally, the obtained products were dried under vacuum at $60{ }^{\circ} \mathrm{C}$ for $24 \mathrm{~h}$.

\subsubsection{Synthesis of $\mathrm{Fe}_{3} \mathrm{O}_{4} @ \mathrm{SiO}_{2}-\mathrm{CH}_{2}=\mathrm{CH}_{2}$}

For vinyl-modification, $\mathrm{Fe}_{3} \mathrm{O}_{4} @ \mathrm{SiO}_{2}(500 \mathrm{mg})$ was dispersed into $200 \mathrm{~mL}$ acetic acid/water $(1: 9, \mathrm{~V} / \mathrm{V})$ in presence of $5 \mathrm{~mL}$ MPS. Next, the mixture was reacted under stirring for $24 \mathrm{~h}$ at room temperature. The products were magnetically separated and rinsed several times with ethanol and ultrapure water. Finally, the products were dried under vacuum at $60{ }^{\circ} \mathrm{C}$ for $12 \mathrm{~h}$.

\subsubsection{Synthesis of Gen-MMIPs and Gen-MNIPs}

Genistein $(1 \mathrm{mmol})$ and 4-VP (4 mmol) were dissolved in a three-necked flask with $40 \mathrm{~mL}$ acetonitrile and stored in the refrigerator for $12 \mathrm{~h}$, then, $20 \mathrm{mmol}$ of EGDMA, 0.12 mmol AIBN, and 200mg $\mathrm{Fe}_{3} \mathrm{O}_{4} @ \mathrm{SiO}_{2}-\mathrm{CH}_{2}=\mathrm{CH}_{2}$ were added into the solution. Next, the mixture was heated to $60^{\circ} \mathrm{C}$ and stirred at $300 \mathrm{rpm}$ under a nitrogen 
atmosphere for $24 \mathrm{~h}$. Gen-MMIPs were collected through magnet separation and washed with methanol/acetic acid solution $(9: 1, \mathrm{v} / \mathrm{v})$ to remove the template until the genistein could not be detected by UV-vis spectrophotometer at $260 \mathrm{~nm}$. Finally, the Gen-MMIPs were dried in a vacuum oven at $60^{\circ} \mathrm{C}$ for $24 \mathrm{~h}$. Fig. 1(A) illustrates the preparation process of Gen-MMIPs.

Gen-MNIPs were prepared with the same condition without adding genistein.

\subsection{Adsorption experiments}

\subsubsection{Kinetic adsorption experiments}

Gen-MMIPs and Gen-MNIPs of $20 \mathrm{mg}$ were added to genistein solution $(20 \mathrm{mg} / \mathrm{L})$ and incubated at different time intervals (0-60 $\mathrm{min})$ at room temperature with constant shaking. The supernatant were separated by an external magnetic field, and the concentration of genistein in the supernatants was measured by HPLC-DAD in $260 \mathrm{~nm}$.

\subsubsection{Isotherms adsorption experiments}

$20 \mathrm{mg}$ Gen-MMIPs or Gen-MNIPs particles were dispersed in a $5 \mathrm{~mL}$ centrifuge tube containing $2.0 \mathrm{~mL}$ genistein solutions of various concentrations within $2-18 \mathrm{mg} / \mathrm{L}$, respectively. The centrifuge tube was shaken for $20 \mathrm{~min}$ at room temperature, the samples were separated by an external magnet. The free concentration of genistein in the supernatant solutions was determined using HPLC-DAD at $260 \mathrm{~nm}$. The adsorption 
amount of genistein on Genistein-MIPs particles (Q) was calculated according to the following eq. (1) [26]; Q tests were performed in triplicate with data reported as the mean values:

$Q_{e}=\left(C_{0}-C_{e}\right) V / m$

where $\mathrm{Q}_{\mathrm{e}}(\mathrm{mg} / \mathrm{g})$ is the equilibrium adsorption capacity; $\mathrm{C}_{0}$ and $\mathrm{C}_{\mathrm{e}}$ are the initial and equilibrium content $(\mathrm{mmol} / \mathrm{L})$ of genistein, respectively; $\mathrm{V}(\mathrm{mL})$ is the volume of genistein solution; $\mathrm{m}(\mathrm{g})$ is the mass of Gen-MMIPs or Gen-MNIPs.

\subsubsection{Selectivity study}

Daidzein was selected as a structural analogue to evaluate the selectivity of GenMMIPs. 20mg MMIPs or MNIPs were added into a $5 \mathrm{~mL}$ mixed solution in which the genistein and daidzein concentrations were $5 \mathrm{mg} / \mathrm{L}$ each, mixtures were shaken at $25^{\circ} \mathrm{C}$ for $20 \mathrm{~min}$. Then, genistein and daidzein in the supernatant were detected using HPLCDAD.

\subsection{Analysis of genistein in real Samples.}

Soy sauce samples were purchased from a local market. The soy sauce samples (2 mL) without spiking or spiked with Genistein standard solutions $(2 \mathrm{~mL} ; 10,20$, or $40 \mathrm{mg} / \mathrm{L})$ were extracted using acetonitrile $(20 \mathrm{~mL})$ for $30 \mathrm{~min}$. Then the solutions were centrifuged and filtered, and the extract solutions were obtained. Genistein- 
MMIPs were added to the extract solution in a $50 \mathrm{ml}$ centrifuge tube. The centrifuge tube was shaken at room temperature for $1 \mathrm{~h}$. A magnet was used to separate Genistein-MMIPs from the solution, and the supernatant were collected in a $50 \mathrm{ml}$ centrifuge tube and measured by HPLC-DAD to determine the amounts of genistein. Then, the genistein was eluted with $3 \mathrm{~mL}$ methanol/acetic acid $(9: 1, \mathrm{v} / \mathrm{v})$ for $30 \mathrm{~min}$, the adsorbent was separated by the magnet. The solution containing template molecule genistein was dissolved in $2 \mathrm{~mL}$ acetonitrile solvent, and the recovery amount of genistein was determined by HPLC-DAD at $260.0 \mathrm{~nm}$. All tests were performed five times with data reported as the mean values.

\section{RESULTS AND DISCUSSION}

\subsection{Characterization}

\subsubsection{FT-IR analysis}

The FT-IR spectra of $\mathrm{Fe}_{3} \mathrm{O}_{4}(\mathrm{a}), \mathrm{Fe}_{3} \mathrm{O}_{4} @ \mathrm{SiO}_{2}(\mathrm{~b}), \mathrm{Fe}_{3} \mathrm{O}_{4} @ \mathrm{SiO}_{2}-\mathrm{CH}_{2}=\mathrm{CH}_{2}(\mathrm{c})$, GenMMIPs(d) were displayed in Fig. 2(A). The peak at $544.0 \mathrm{~cm}^{-1}$ characterized $\mathrm{Fe}-\mathrm{O}$ stretching vibration of $\mathrm{Fe}_{3} \mathrm{O}_{4}$ nanoparticles, in comparison with the curve of $\mathrm{Fe}_{3} \mathrm{O}_{4}$, the new characteristic peaks at $1088 \mathrm{~cm}^{-1}$ was attributed to the $\mathrm{Si}-\mathrm{O}$ stretching vibration, indicated that $\mathrm{SiO}_{2}$ was coated on the surface of $\mathrm{Fe}_{3} \mathrm{O}_{4}$ nanoparticles. The absorption band at $1632 \mathrm{~cm}^{-1}$ demonstrated the existence of $-\mathrm{C}=\mathrm{C}$, which proved that vinyl was 
synthesized on the surface of $\mathrm{Fe}_{3} \mathrm{O}_{4} @ \mathrm{SiO}_{2}$. The absorption band at $1710 \mathrm{~cm}^{-1}$ and 2922 $\mathrm{cm}^{-1}$ were due to the stretching vibration of $\mathrm{C}=\mathrm{O}$ and $\mathrm{C}-\mathrm{H}$ in EGDMA, respectively[27], which confirms that the MMIPs layer was polymerized on the surface of the $\mathrm{Fe}_{3} \mathrm{O}_{4} @ \mathrm{SiO}_{2}-\mathrm{CH}_{2}=\mathrm{CH}_{2}$.

\subsubsection{Evaluation of magnetism}

The magnetic properties of the materials were characterized by vibratory sample magnetometer (VSM). Magnetic intensity of the synthesized materials decreased with the increase of the polymers shell structure (Fig.2 B). Four magnetic hysteresis loops are all symmetrical to the origin, indicating the materials have superparamagnetic[28]. The saturation magnetization of $\mathrm{Fe}_{3} \mathrm{O}_{4}, \mathrm{Fe}_{3} \mathrm{O}_{4} @ \mathrm{SiO}_{2}, \mathrm{Fe}_{3} \mathrm{O}_{4} @ \mathrm{SiO}_{2}-\mathrm{CH}_{2}=\mathrm{CH}_{2}, \mathrm{Gen}-$ MMIPs were 63.92, 48.77, 41.62, 24.79 emug $^{-1}$, respectively. Gen-MMIPs can homogeneously disperse in aqueous solutions and can be separated rapidly in solution by magnetic field. (Illustration in Fig.2 B)

\subsubsection{TEM characterization}

The morphology of $\mathrm{Fe}_{3} \mathrm{O}_{4}, \mathrm{Fe}_{3} \mathrm{O}_{4} @ \mathrm{SiO}_{2}, \mathrm{Fe}_{3} \mathrm{O}_{4} @ \mathrm{SiO}_{2}-\mathrm{CH}_{2}=\mathrm{CH}_{2}$, Gen-MMIPs were characterized by TEM. The diameters of $\mathrm{Fe}_{3} \mathrm{O}_{4}$ nanoparticles (Fig. 3A), $\mathrm{Fe}_{3} \mathrm{O}_{4} @ \mathrm{SiO}_{2}$ (Fig. 3B), $\mathrm{Fe}_{3} \mathrm{O}_{4} @ \mathrm{SiO}_{2}-\mathrm{CH}_{2}=\mathrm{CH}_{2}$ (Fig. 3C) and Gen-MMIPs (Fig. 3D) were about 10, 20, 50 and 500 nm, respectively. Compared with $\mathrm{Fe}_{3} \mathrm{O}_{4}$ and $\mathrm{Fe}_{3} \mathrm{O}_{4} @ \mathrm{SiO}_{2}$, 
$\mathrm{Fe}_{3} \mathrm{O}_{4} @ \mathrm{SiO}_{2}-\mathrm{CH}_{2}=\mathrm{CH}_{2}$ have larger area and more layers of imprinted, which indicated that vinyl is modified on the surface of $\mathrm{Fe}_{3} \mathrm{O}_{4}$. After MIPs were coated, the volume of Gen-MMIPs was increased significantly, and the morphologies of the Gen-MMIPs had a uniform distribution of core-shell structure. The core-shell structure of Gen-MMIPs were beneficial for the mass transfer between the solution and the surface of GenMMIPs.

\subsubsection{SEM characterization}

It can be seen from Fig. $3 \mathrm{E}$ that the size of $\mathrm{Fe}_{3} \mathrm{O}_{4}$ is nanometer and evenly dispersed, compared with the $\mathrm{Fe}_{3} \mathrm{O}_{4}$ particles. Hydrolytic TEOS coat on the surface of the $\mathrm{Fe}_{3} \mathrm{O}_{4}$ to form smoother particles (Fig. 3F). Both Gen-MMIPs and Gen-MNIPs exhibited relatively spherical structure and good dispersibility, which would provide high specific surface area and superior structure to facilitate mass transfer of genistein.

The Gen-MNIPs micrograph showed less porous surface, it was attributed to non-use of the template during the polymerization process (Fig. 3G). Compared to Gen-MNIPs, Gen-MMIPs revealed a porous morphology and possessed more binding sites, which is beneficial for the adsorption and release of template molecules (Fig. 3H). 


\subsection{Adsorption experiments}

\subsubsection{Adsorption kinetics}

The adsorption kinetic curve of genistein absorbed on Gen-MMIPs and Gen-

MNIPs were displayed in Fig. 4A, which showed the adsorption capacity increased with the increase of adsorption time and reached balance at about $20 \mathrm{~min}$, therefore, 20 min was selected as optimum adsorption time because the recognition sites on the surface of Gen-MMIPs were occupied completely. The maximum adsorption amounts of Gen-MMIPs and Gen-MNIPs are 0.74 and $0.21 \mathrm{mg} / \mathrm{g}$, respectively.

\subsubsection{Adsorption isotherms}

The isothermal adsorption curves of Gen-MMIPs and Gen-MNIPs for genistein were estimated for different genistein concentrations $(1-18 \mathrm{mg} / \mathrm{L})$. As illustrated in Fig. 4B, when the concentrations of genistein was below $12 \mathrm{mg} / \mathrm{L}$, the adsorption capacity increased obviously with the increase of the concentrations of genistein. The adsorption capacity became saturated when the concentration of genistein exceeded $12 \mathrm{mg} / \mathrm{L}$. The equilibrium adsorption capacity of genistein on Gen-MMIPs was 1.55 $\mathrm{mg} / \mathrm{g}, 2.8$ times higher than that of Gen-MNIPs $(0.55 \mathrm{mg} / \mathrm{g})$. It could be seen that the adsorption capacity for genistein of Gen-MMIPs was much higher than that of GenMNIPs at the same initial concentration. The results indicated that the Gen-MMIPs 
possess more imprinted sites on the surface, which proved that Gen-MMIPs were more specific to genistein than Gen-MNIPs.

The Scatchard equation was adopted to assessment the binding ability of the Gen-MMIPs and Gen-MNIPs. The Scatchard equation was expressed as follows eqs.

(2) and (3), respectively[29].

$Q_{e} / C_{e}=\left(Q_{m}-Q_{e}\right) / K_{d}$

$K_{a}=1 / K_{d}$

where $\mathrm{Q}_{\mathrm{e}}$ was the equilibrium adsorption capacity $(\mathrm{mg} / \mathrm{g}), \mathrm{C}_{\mathrm{e}}$ was the genistein concentration at equilibrium $(\mathrm{mg} / \mathrm{mL}), \mathrm{Q}_{\mathrm{m}}$ was the apparent maximum binding amount (mg/g), $\mathrm{K}_{d}$ was the equilibrium dissociation constant $(\mathrm{g} / \mathrm{L})$, and $\mathrm{K}_{\mathrm{a}}$ was the equilibrium binding constant $(\mathrm{L} / \mathrm{g})$. As shown in Fig. 4C, the Scatchard plot for the Gen-MMIPs was one straight line, which indicated that there was one type of binding site in Gen-MMIPs. According to the linear regression equation, the $\mathrm{K}_{\mathrm{a}}$ of GenMMIPs and Gen-MNIPs were calculated to be $0.0684 \mathrm{~L} / \mathrm{g}$ and $0.0596 \mathrm{~L} / \mathrm{g}$, respectively, which indicated the affinity of the Gen-MMIPs to genistein is higher than that of Gen-MNIPs.

\subsubsection{Selectivity of the Gen-MMIPs}

To investigate the selectivity of Gen-MMIPs towards genistein, daidzein was 
selected as a potential structural analog, As shown in Fig. 5A. The adsorption capacities of Gen-MMIPs and Gen-MNIPs for genistein and daidzein were displayed in Fig. 5B. It was obvious that the adsorption capacity of Gen-MMIPs for the genistein was much higher than that of Gen-MNIPs. The adsorption capacity of GenMIPs for genistein $(2 \mathrm{mg} / \mathrm{g})$ was about 4 times than that of daidzein $(0.5 \mathrm{mg} / \mathrm{g})$, which proved that Gen-MMIPs could possess more surface imprinted sites for recognition of genistein.

As shown in Fig. 5B, there was no obvious adsorption difference of Gen-MNIPs for genistein and daidzein, which suggested that Gen-MNIPs did not have the specific recognition sites that existed on their surface. Additionally, the selectivity properties of Gen-MMIPs and Gen-MNIPs towards genistein were further evaluated by imprinting factor $(\alpha)$ and selectivity factor $(\beta)[29,30]$, which were calculated according to eqs (4) and (5), respectively.

$\alpha=Q_{M I P} / Q_{N I P}$

$\beta=\alpha_{G e n} / \alpha_{D a d}$

where $Q_{\text {MIP }}$ and $Q_{\text {NIP }}(\mathrm{mg} / \mathrm{g})$ were the adsorption capacity of Gen-MMIPs and

Gen-MNIPs, respectively, $\alpha_{\text {Gen }}$ and $\alpha_{\text {Dad }}$ factors were the imprinting factor for template (genistein) and analog (daidzein). The imprinting factors $(\alpha)$ were calculated 
as 2.81 and 1.35 for genistein and daidzein, respectively. The selectivity factor $(\beta)$

was calculated as 2.08 . The results demonstrated that the obtained Gen-MMIPs

exhibited good selectivity for genistein.

\subsubsection{Reusability}

The reusability of molecularly imprinted polymers was an important property for application. Reusability of the Gen-MMIPs was depicted in Fig. 5C. Compared with the initial adsorption capacity, after ten adsorption-elution cycles, the adsorption capacity of Gen-MMIPs was $85 \%$ of the initial. It was attributed to the imprinted sites of the Gen-MMIPs were very stable which were not destroyed during the process of adsorption-elution cycles.

\subsection{Analysis of spiked food samples}

To check the applicability of the synthesized Gen-MMIPs in real sample analysis using HPLC-DAD, Soy sauce samples were pretreated according to the method in Section 2.5. The procedures used for Gen-MMIPs are shown in Fig. 1(B).

\subsubsection{Analytical validation}

Basing on Signal-to-noise (S/N), the limit of detection (LOD) as 3 times of $(\mathrm{S} / \mathrm{N})$ and the limit of quantification (LOQ) as 10 times of $(\mathrm{S} / \mathrm{N})$ were calculated, which were $0.39 \mathrm{mg} / \mathrm{L}$ and $1.3 \mathrm{mg} / \mathrm{L}$. Recovery and relative standard deviation (RSD) were 
assessed at three levels. The results are presented in Table 1.

\subsubsection{Chromatograms of actual samples}

To further validate the performance of Gen-MMIPs, the real samples spiked with genistein were assessed by applying the optimum MMIPs-HPLC-DAD method.

The spiked sample was shown in Figure 6, besides the absorption peak of genistein is at $11 \mathrm{~min}$, more than 10 peaks were observed, which indicates that the actual sample was a complex matrix, and genistein accounts for a small proportion. While after enrichment by the Gen-MMIPs, the peaks of genistein were observed without significant change, but the peaks of impurities decrease sharply. Therefore, the results show that, most of impurities were successfully removed, but there are still a few impurities in the solution after enrichment, which is caused by the physical adsorption of Gen-MMIPs. The recovery of the spiked sample for genistein is $85.7 \%-88.5 \%$, which demonstrated that Gen-MMIPs had excellent specificity for genistein and could extract genistein in actual samples.

\section{CONCLUSIONS}

In this study, a new adsorbent material of Gen-MMIPs for genistein was successfully synthesized by surface molecular imprinting. The obtained Gen-MMIPs were characterized by SEM, TEM, FT-IR and VSM. The adsorption isotherms were investigated and binding characteristics were found to match the Scatchard models, 
the Gen-MMIPs showed higher molecular selectivity and adsorption capacity than Gen-MNIPs, the kinetics adsorption of Gen-MMIPs reached the adsorption equilibrium in $20 \mathrm{~min}$. Superparamagnetic ensured that Gen-MMIPs could be rapidly separated by applying an external magnetic field without tedious centrifugation or filtration. In addition, Gen-MMIPs had excellent reusability. The obtained GenMMIPs could selectively recognize and effectively extract genistein from soy sauce samples with satisfactory recoveries and reproducibility. This work provided a new idea for the pretreatment method of genistein and has important practical value for the separation, analysis and application of genistein.

\section{Compliance with Ethical Standards}

Conflict of interest The authors declare that they have no conflict of interest.

\section{References:}

[1] Vodnik VV, Mojić M, Stamenović U, Otoničar M, Ajdžanović V, Maksimović-Ivanić D, Mijatović

S, Marković MM, Barudžija T, Filipović B, Milošević V, Šošić-Jurjević B (2021) Materials Science and Engineering: C 124:112078

[2] Ishimi Y, Miyaura C, Ohmura M, Onoe Y, Sato T, Uchiyama Y, Ito M, Wang X, Suda T, Ikegami S (1999) ENDOCRINOLOGY 140:1893-1900

[3] de Gregorio C, Marini H, Alibrandi A, Di Benedetto A, Bitto A, Adamo E, Altavilla D, Irace C, Di Vieste G, Pancaldo D, Granese R, Atteritano M, Corrao S, Licata G, Squadrito F, Arcoraci V (2017) NUTRIENTS 9:584

[4] Saha S, Kroon PA (2020) J ANAL METHODS CHEM 2020:1-9

[5] Nalewajko-Sieliwoniuk E, Hryniewicka M, Jankowska D, Kojło A, Kamianowska M, Szczepański M (2020) FOOD CHEM 327:126996

[6] Nalewajko-Sieliwoniuk E, Hryniewicka M, Jankowska D, Kojło A, Kamianowska M, Szczepański 
M (2020) FOOD CHEM 327:126996

[7] Ferrer I, Barber LB, Thurman EM (2009) J CHROMATOGR A 1216:6024-6032

[8] Sakamoto S, Yusakul G, Pongkitwitoon B, Paudel MK, Tanaka H, Morimoto S (2015) FOOD CHEM $169: 127-133$

[9] Aresta A, Cotugno P, Massari F, Zambonin C (2017) J FOOD QUALITY 2017:1-5

[10] Aresta A, Di Grumo F, Zambonin C (2016) FOOD ANAL METHOD 9:925-933

[11] Nalewajko-Sieliwoniuk E, Hryniewicka M, Jankowska D, Kojło A, Kamianowska M, Szczepański M (2020) FOOD CHEM 327:126996

[12] ARAUJO J, SILVA M, CHAVES J (2007) FOOD CHEM 105:266-272

[13] Klejdus B, Mikelová R, Petrlová J, Potěšil D, Adam V, Stiborová M, Hodek P, Vacek J, Kizek R, Kubáň V (2005) J CHROMATOGR A 1084:71-79

[14] Chen L, Wang X, Lu W, Wu X, Li J (2016) CHEM SOC REV 45:2137-2211

[15] Mayes AG, Mosbach K (1996) Analytical chemistry (Washington) 68:3769-3774

[16] Ye L, Mosbach K (2008) CHEM MATER 20:859-868

[17] SPIVAK D (2005) ADV DRUG DELIVER REV 57:1779-1794

[18] Smith RM (2003) J CHROMATOGR A 1000:3-27

[19] Wierucka M, Biziuk M (2014) TrAC Trends in Analytical Chemistry 59:50-58

[20] Zhang Y, Liu R, Hu Y, Li G (2009) ANAL CHEM 81:967-976

[21] Liu K, Lin H, Thomas JL, Shih Y, Chen J, Lee M (2021) IND CROP PROD 163:113291

[22] Li X, Zhu G, Luo Y, Yuan B, Feng Y (2013) TrAC Trends in Analytical Chemistry 45:233-247

[23] Song H, Zhang H, He Y, Gao R, Wang Y, Wang W, Pfefferle LD, Tang X, Tang Y (2021) FOOD CHEM 356:129722

[24] Chen S, Fu J, Li Z, Zeng Y, Li Y, Su X, Jiang X, Yang H, Huang L, Zou L, He L, Liu S, Ao X, Yang Y (2019) J CHROMATOGR A 1602:124-134

[25] Dinali LAF, de Oliveira HL, Teixeira LS, Da Silva ATM, D'Oliveira KA, Cuin A, Borges KB (2020) MICROCHEM J 154:104648 
[26] Ning F, Peng H, Li J, Chen L, Xiong H (2014) J AGR FOOD CHEM 62:7436-7443

[27] Liu Z, Wang X, Chen J, Gao J, Yu S, Wang X (2020) MICROCHEM J 157:105052

[28] Zhao Q, Zhao H, Yang X, Zhang H, Dong A, Wang J, Li B (2018) J CHROMATOGR A 1572:9-

19

[29] Ma X, Lin H, He Y, She Y, Wang M, Abd El-Aty AM, Afifi NA, Han J, Zhou X, Wang J, Zhang J (2019) J CHROMATOGR A 1598:39-48

[30] Cheng Y, Nie J, Liu H, Kuang L, Xu G (2020) J CHROMATOGR A 1630:461531 


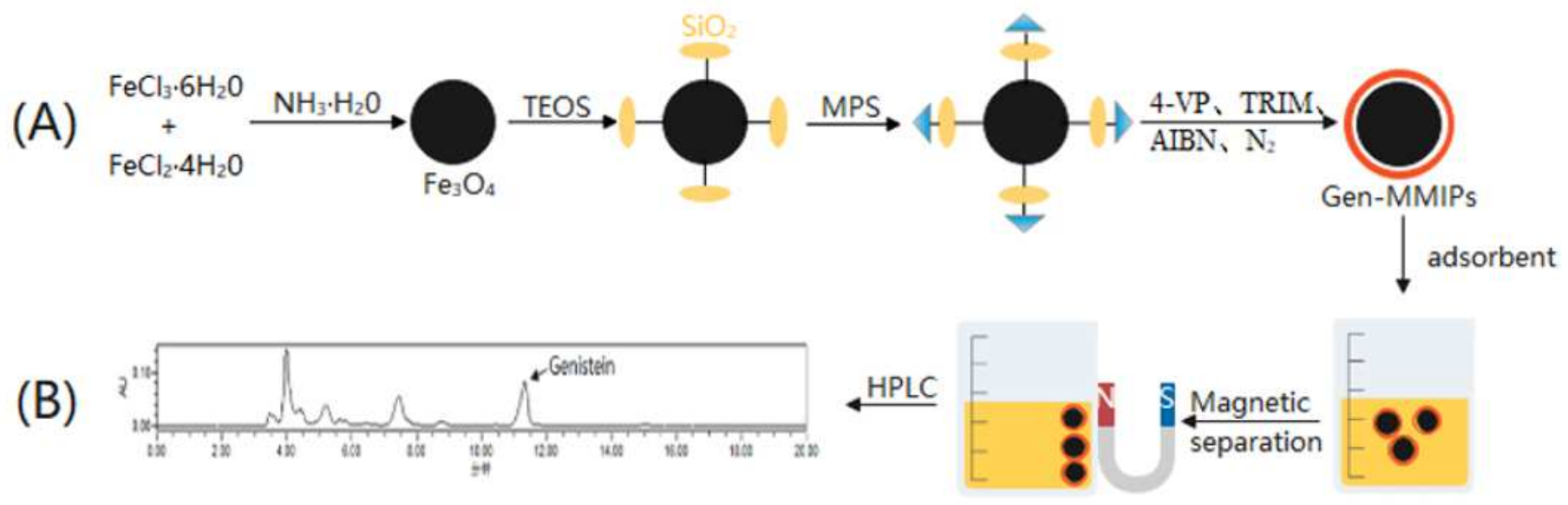

Figure 1

Synthesis and detection of Gen-MMIPs
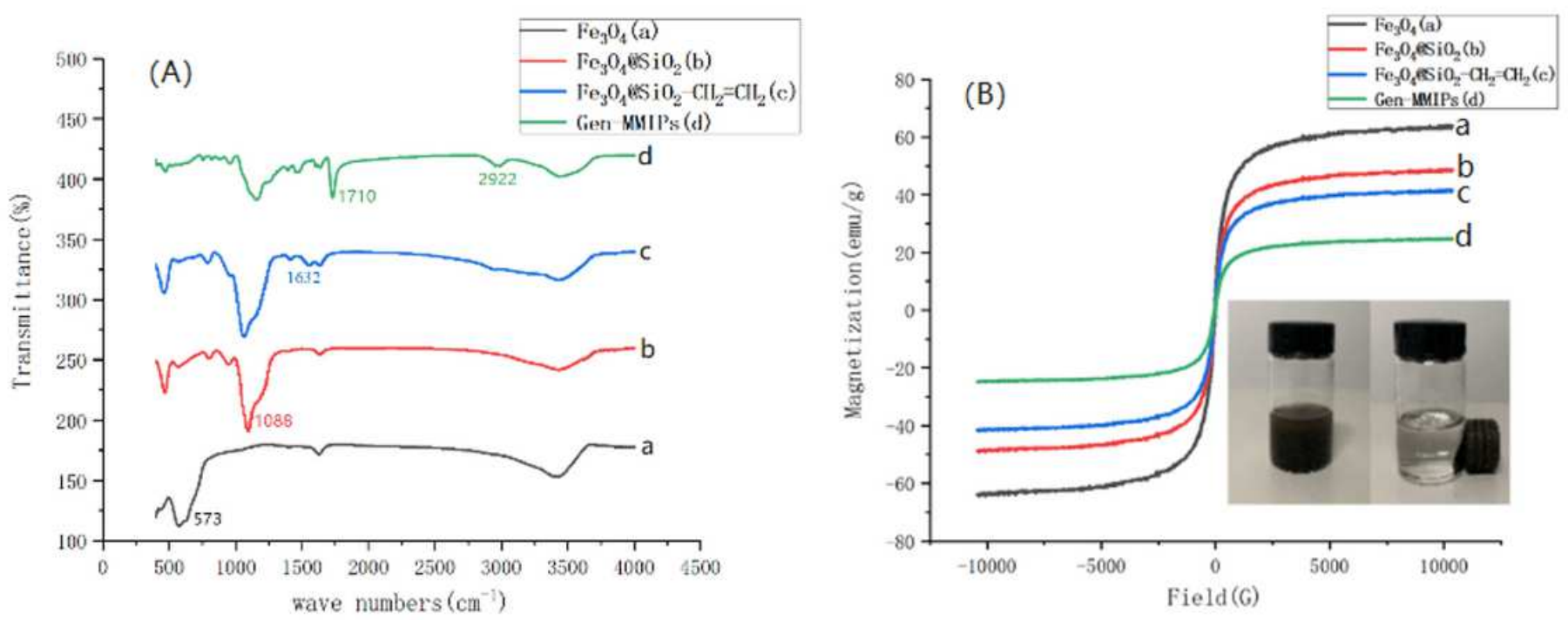

Figure 2

(A) FT-IR spectra of Fe304(a), Fe304@SiO2(b), Fe304@SiO2-CH2=CH2(c), Gen-MIPs(d). (B) VSM of Fe304(a),Fe304@SiO2(b), Fe3O4@SiO2-CH2=CH2(c), Gen-MMIPs(d) 


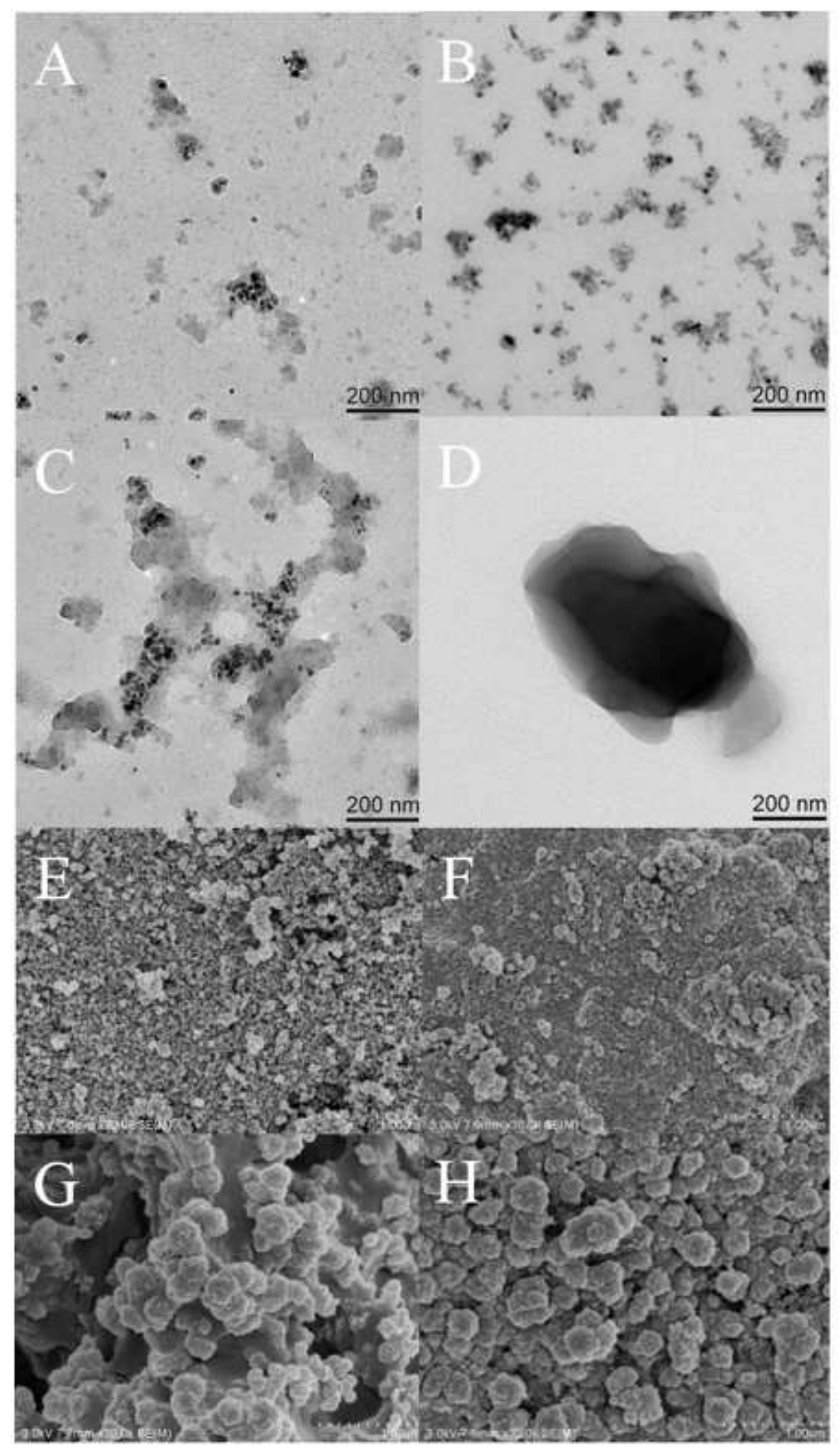

\section{Figure 3}

TEM images of the Fe3O4 (A), Fe3O4@SiO2 (B), Fe304@SiO2-CH2=CH2 (C) and Gen-MMIPs (D). SEM images of the Fe3O4 (E), Fe304@SiO2 (F), Gen-MNIPs (G) and Gen-MMIPs(H) 

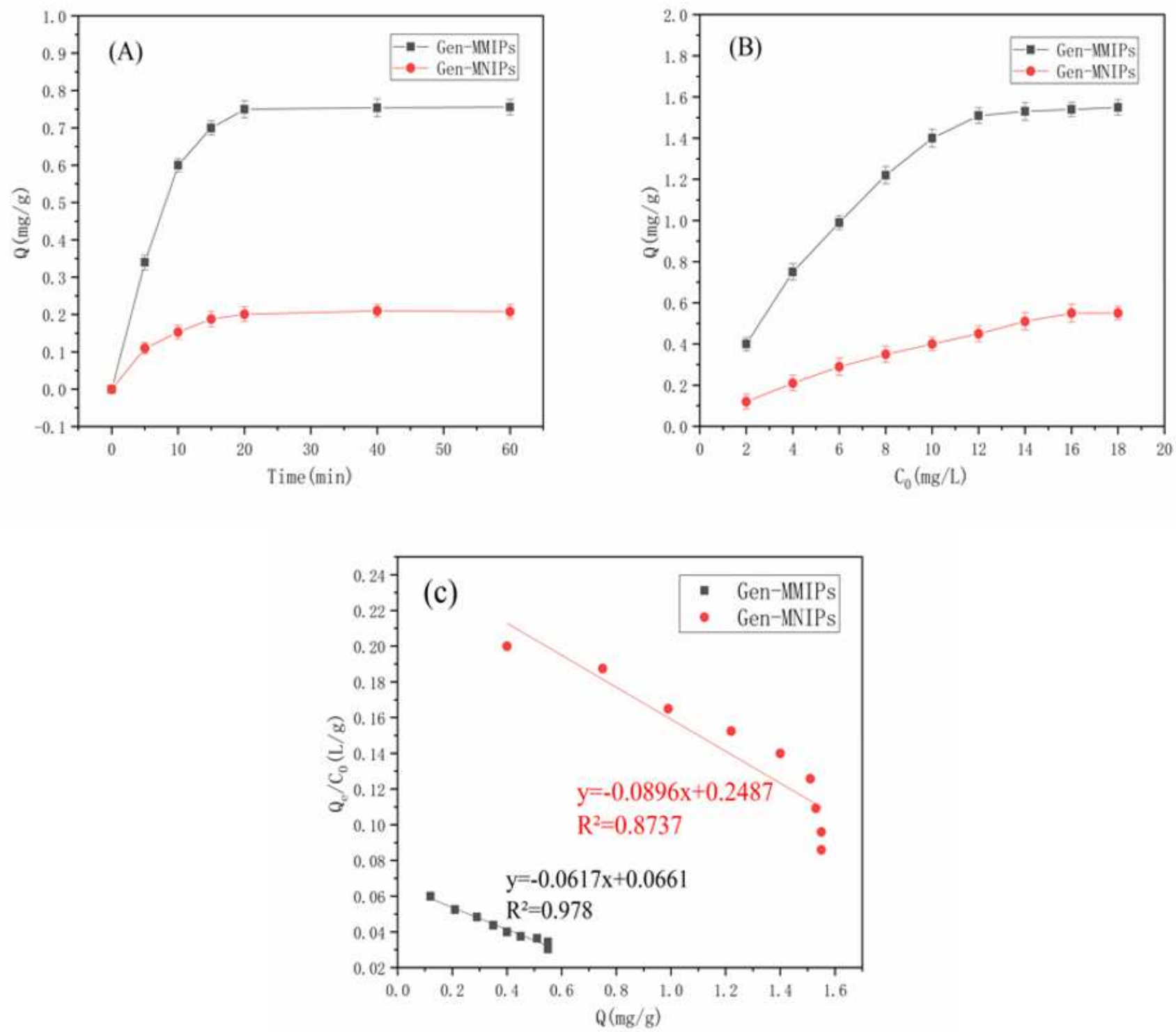

Figure 4

(A) Adsorption kinetic of Genistein on Gen-MMIPs and Gen-MNIPs, (B) adsorption isotherms of Genistein on Gen-MMIPs and Gen-MNIPs, and (C) Scatchard plots of Gen-MMIPs and Gen-MNIPs 

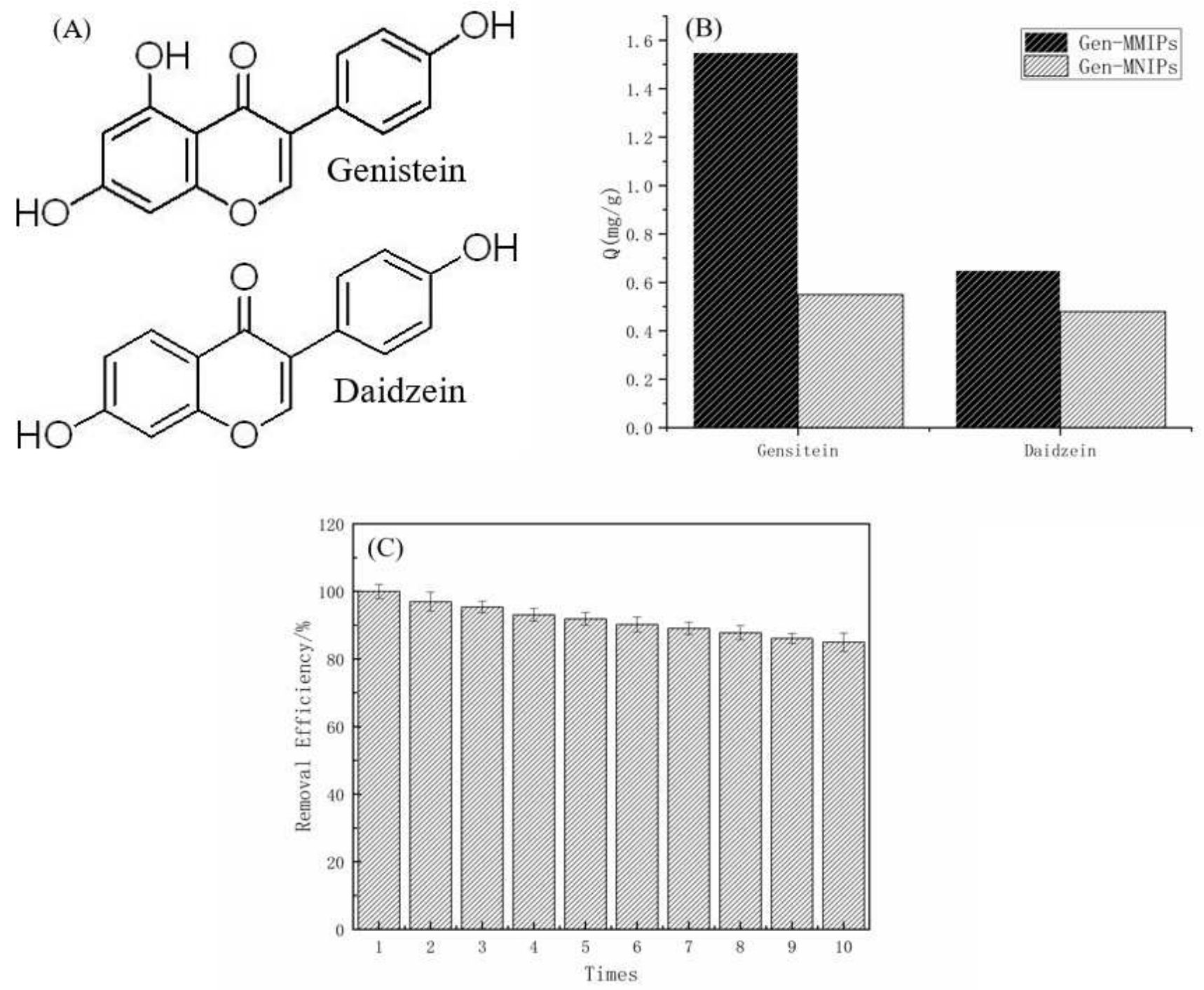

Figure 5

The structures of genistein and daidzein, (B) selective adsorption of Gen-MMIPs and Gen-MNIPs for genistein and daidzein. (C) Adsorption of Genistein by Gen-MMIPs in ten adsorption-desorption cycles 


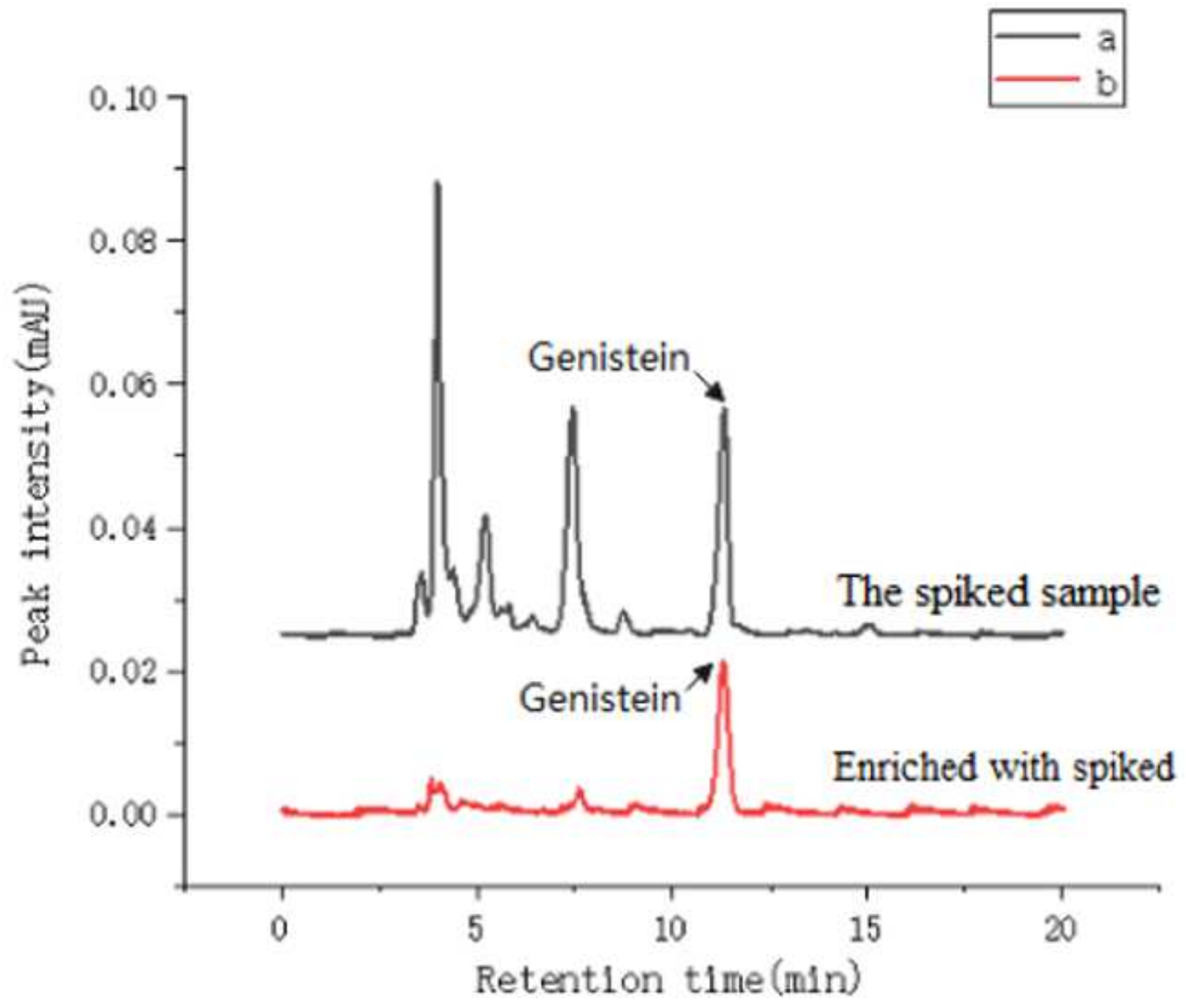

Figure 6

HPLC chromatograms of spiked and enriched with spiked 